\title{
On complete intersection toric ideals of graphs
}

\author{
Christos Tatakis • Apostolos Thoma
}

Received: 27 May 2012 / Accepted: 5 October 2012 / Published online: 18 October 2012

(C) Springer Science+Business Media New York 2012

\begin{abstract}
We study the graphs $G$ for which their toric ideals $I_{G}$ are complete intersections. In particular, we prove that for a connected graph $G$ such that $I_{G}$ is a complete intersection all of its blocks are bipartite except for at most two. We prove that toric ideals of graphs which are complete intersections are circuit ideals. In this case, the generators of the toric ideal correspond to even cycles of $G$ except of at most one generator, which corresponds to two edge disjoint odd cycles joint at a vertex or with a path. We prove that the blocks of these graphs satisfy the odd cycle condition. Finally, we characterize all complete intersection toric ideals of graphs which are normal.
\end{abstract}

Keywords Complete intersections · Graphs · Toric ideals

\section{Introduction}

The complete intersection property of the toric ideals of graphs was first studied by L. Doering and T. Gunston in [4]. In 1998, A. Simis proved that for a bipartite graph $G$ for which the toric ideal $I_{G}$ is a complete intersection the number of chordless cycles of $G$ is equal to the number $m-n+r$, where $m$ is the number of edges, $n$ the number of vertices, and $r$ the number of connected components of the graph $G$; see [19]. The following year, M. Katzman proved that for a bipartite graph $G$ the corresponding ideal $I_{G}$ is a complete intersection if and only if any two chordless cycles have at most one edge in common; see [11]. Finally I. Gitler, E. Reyes, and R. Villarreal completely determined the form of the bipartite graphs for which the

C. Tatakis · A. Thoma ( $\varangle)$

Department of Mathematics, University of Ioannina, Ioannina 45110, Greece

e-mail: athoma@uoi.gr

C. Tatakis

e-mail: chtataki@cc.uoi.gr 
Fig. 1 A planar complete intersection graph

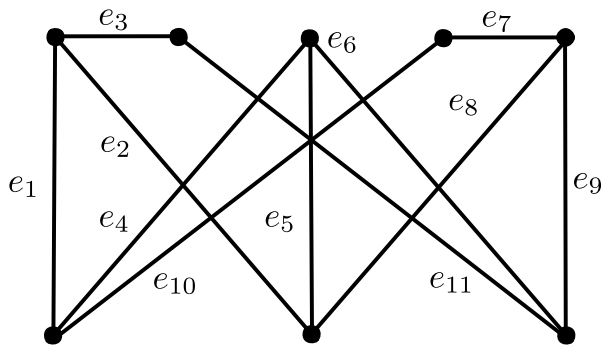

toric ideal $I_{G}$ is a complete intersection; see [5, 6]. They are the ring graphs. Given a graph $H$, we call a path $P$ an $H$-path if $P$ is non-trivial and meets $H$ exactly in its ends. A graph $G$ is a ring graph if each block of $G$ which is not an edge or a vertex can be constructed from a cycle by successively adding $H$-paths of length at least two that meet graphs $H$ already constructed in two adjacent vertices.

Theorem 1.1 (I. Gitler, E. Reyes, and R. Villarreal [6]) If $G$ is a bipartite graph then $I_{G}$ is a complete intersection if and only if $G$ is a ring graph.

In this article, we try to characterize complete intersection toric ideals of a general simple graph. Note that it is enough to answer the problem for a connected graph, since for the toric ideal of a graph $G$ to be a complete intersection it is enough that for every connected component $G^{\prime}$ of $G$ the ideal $I_{G^{\prime}}$ is a complete intersection, and conversely. In this article, we will assume that all graphs considered are connected, except if stated otherwise.

The situation for a general graph is much more complicated than the case of a bipartite graph. For example, bipartite complete intersection graphs are always planar, see $[6,11]$, but this is not the general case as the following example shows, see also [11].

Let $G$ be the graph with 11 edges and 8 vertices in Fig. 1 . The height of the toric ideal $I_{G}$ is three, see [23], and $I_{G}$ is generated by the binomials $e_{1} e_{5}-e_{2} e_{4}, e_{5} e_{9}-$ $e_{6} e_{8}, e_{3} e_{9} e_{10}-e_{1} e_{7} e_{11}$, therefore it is a complete intersection. The graph $G$ is a subdivision of $K_{3,3}$ and therefore it is not planar, see [12]. A subdivision of a graph $G$ is any graph that can be obtained from $G$ by replacing edges by paths.

Note also that the ideal of a general graph is much more complicated than the ideal of a bipartite graph. The generators of the toric ideal of a bipartite graph correspond to chordless even cycles of the graph. While the generators of the general graph have a more complicated structure, see Theorems 2.1, 2.7. The fact that the generators of a complete intersection toric ideal are very simple is very interesting, all of them correspond to even cycles with at most one exemption, see Theorem 5.4. Actually, this is one of the properties that characterize complete intersection toric ideals of graphs, see Theorem 5.5.

In the second section, we review several notions from graph theory that will be useful in the sequel. We define the toric ideal of a graph and we recall several results about the elements of the Graver basis, the circuits and the elements of a minimal system of generators of the toric ideal of the graph. The third section contains basic 
results about complete intersections toric ideals of graphs. The fourth section contains one of the main results of the article that in a graph $G$ for which the toric ideal $I_{G}$ is a complete intersection either all blocks are bipartite, or all blocks are bipartite except for one, or all blocks are bipartite except for two. In the case that there are exactly two non-bipartite blocks, they have a special position in the graph, the two blocks are contiguous. The fifth section contains the result that complete intersection toric ideals are circuit ideals and give a necessary and sufficient condition for a graph $G$ to be a complete intersection. The final section proves that biconnected complete intersections graphs satisfy the odd cycle condition and gives a necessary and sufficient condition for the edge ring of a complete intersection graph to be normal.

In the same problem, independently from us, I. Bermejo, I. García-Marco and E. Reyes are working on providing combinatorial and algorithmic characterizations of general graphs such that their toric ideals are complete intersections; see [1].

\section{Toric ideals of graphs}

Let $A=\left\{\mathbf{a}_{1}, \ldots, \mathbf{a}_{m}\right\} \subseteq \mathbb{N}^{n}$ be a vector configuration in $\mathbb{Q}^{n}$ and $\mathbb{N} A:=\left\{l_{1} \mathbf{a}_{1}+\cdots+\right.$ $\left.l_{m} \mathbf{a}_{m} \mid l_{i} \in \mathbb{N}\right\}$ the corresponding affine semigroup. We grade the polynomial ring $K\left[x_{1}, \ldots, x_{m}\right]$ over any field $K$ by the semigroup $\mathbb{N} A$ setting $\operatorname{deg}_{A}\left(x_{i}\right)=\mathbf{a}_{i}$ for $i=$ $1, \ldots, m$. For $\mathbf{u}=\left(u_{1}, \ldots, u_{m}\right) \in \mathbb{N}^{m}$, we define the $A$-degree of the monomial $\mathbf{x}^{\mathbf{u}}:=$ $x_{1}^{u_{1}} \cdots x_{m}^{u_{m}}$ to be

$$
\operatorname{deg}_{A}\left(\mathbf{x}^{\mathbf{u}}\right):=u_{1} \mathbf{a}_{1}+\cdots+u_{m} \mathbf{a}_{m} \in \mathbb{N} A .
$$

The toric ideal $I_{A}$ associated to $A$ is the prime ideal generated by all the binomials $\mathbf{x}^{\mathbf{u}}-\mathbf{x}^{\mathbf{v}}$ such that $\operatorname{deg}_{A}\left(\mathbf{x}^{\mathbf{u}}\right)=\operatorname{deg}_{A}\left(\mathbf{x}^{\mathbf{v}}\right)$, see [22]. For such binomials, we define $\operatorname{deg}_{A}\left(\mathbf{x}^{\mathbf{u}}-\mathbf{x}^{\mathbf{v}}\right):=\operatorname{deg}_{A}\left(\mathbf{x}^{\mathbf{u}}\right)$.

Let $G$ be a simple finite connected graph on the vertex set $V(G)=\left\{v_{1}, \ldots, v_{n}\right\}$ and let $E(G)=\left\{e_{1}, \ldots, e_{m}\right\}$ be the set of edges of $G$. We denote by $K\left[e_{1}, \ldots, e_{m}\right]$ the polynomial ring in the $m$ variables $e_{1}, \ldots, e_{m}$ over a field $K$. We will associate each edge $e=\left\{v_{i}, v_{j}\right\} \in E(G)$ with $a_{e}=v_{i}+v_{j}$ in the free abelian group generated by the vertices of $G$ and let $A_{G}=\left\{a_{e} \mid e \in E(G)\right\}$. We denote by $I_{G}$ the toric ideal $I_{A_{G}}$ in $K\left[e_{1}, \ldots, e_{m}\right]$ and by $\operatorname{deg}_{G}$ the $\operatorname{deg}_{A_{G}}$. By $K[G]$ we denote the subalgebra of $K\left[v_{1}, \ldots, v_{n}\right]$ generated by all quadratic monomials $v_{i} v_{j}$ such that $e=\left\{v_{i}, v_{j}\right\} \in$ $E(G) . K[G]$ is an affine semigroup ring and it is called the edge ring of $G$.

A cut vertex (respectively, cut edge) is a vertex (respectively, edge) of the graph whose removal increases the number of connected components of the remaining subgraph. A graph is called biconnected if it is connected and does not contain a cut vertex. A block is a maximal biconnected subgraph of a given graph $G$.

A walk of length $s$ connecting $v_{1} \in V(G)$ and $v_{s+1} \in V(G)$ is a finite sequence of the form

$$
w=\left(\left\{v_{1}, v_{2}\right\},\left\{v_{2}, v_{3}\right\}, \ldots,\left\{v_{s}, v_{s+1}\right\}\right)
$$

with each $e_{j}=\left\{v_{j}, v_{j+1}\right\} \in E(G), 1 \leq j \leq s$. An even (respectively, odd) walk is a walk of even (respectively, odd) length. The walk $w$ is called closed if $v_{s+1}=v_{1}$. 
We call a walk $w^{\prime}=\left(e_{j_{1}}, \ldots, e_{j_{t}}\right)$ a subwalk of $w$ if $e_{j_{1}} \cdots e_{j_{t}} \mid e_{1} \cdots e_{s}$. A cycle is a closed walk

$$
\left(\left\{v_{1}, v_{2}\right\},\left\{v_{2}, v_{3}\right\}, \ldots,\left\{v_{s}, v_{1}\right\}\right)
$$

with $v_{i} \neq v_{j}$, for every $1 \leq i<j \leq s$. For convenience, by $\mathbf{w}$ we denote the subgraph of $G$ with vertices the vertices of the walk and edges the edges of the walk $w$. Given an even closed walk

$$
w=\left(e_{i_{1}}, \ldots, e_{i_{2 q-1}}, e_{i_{2 q}}\right)
$$

of the graph $G$, we denote by

$$
E^{+}(w)=\prod_{k=1}^{q} e_{i_{2 k-1}}=\mathbf{e}^{w^{+}}, \quad E^{-}(w)=\prod_{k=1}^{q} e_{i_{2 k}}=\mathbf{e}^{w^{-}},
$$

and by $B_{w}$ the binomial

$$
B_{w}=\prod_{k=1}^{q} e_{i_{2 k-1}}-\prod_{k=1}^{q} e_{i_{2 k}}
$$

belonging to the toric ideal $I_{G}$; also by $w^{+}, w^{-}$we denote the exponent vectors of the monomials $E^{+}(w), E^{-}(w)$ and by $\mathbf{w}^{+}, \mathbf{w}^{-}$the sets $\left\{e_{i_{1}}, e_{i_{3}}, \ldots, e_{i_{2 q-1}}\right\}$, $\left\{e_{i_{2}}, e_{i_{4}}, \ldots, e_{i_{2 q}}\right\}$, respectively. Actually, the toric ideal $I_{G}$ is generated by binomials of this form, see [23]. An even closed walk $w=\left(e_{i_{1}}, \ldots, e_{i_{2 q-1}}, e_{i_{2 q}}\right)$ is said to be primitive if there exists no even closed subwalk $\xi$ of $w$ of smaller length such that $E^{+}(\xi) \mid E^{+}(w)$ and $E^{-}(\xi) \mid E^{-}(w)$.

Every even primitive walk $w=\left(e_{i_{1}}, \ldots, e_{i_{2 q}}\right)$ partitions the set of edges of $w$ in the two sets $\mathbf{w}^{+}=\left\{e_{i_{j}} \mid j\right.$ odd $\}$ and $\mathbf{w}^{-}=\left\{e_{i_{j}} \mid j\right.$ even $\}$, otherwise if $e_{i_{k}} \in \mathbf{w}^{+} \cap \mathbf{w}^{-}$then for the even closed subwalk $\xi=\left(e_{i_{k}}, e_{i_{k}}\right)$ we have $E^{+}(\xi) \mid E^{+}(w)$ and $E^{-}(\xi) \mid E^{-}(w)$. The edges of $\mathbf{w}^{+}$are called odd edges of the walk and those of $\mathbf{w}^{-}$are called even. A sink of a block $\mathcal{B}$ of the graph $\mathbf{w}$ is a common vertex of two odd or two even edges of the walk $w$ which belong to the block $\mathcal{B}$.

A necessary and sufficient characterization of the primitive walks of a graph, were given by E. Reyes, Ch. Tatakis and A. Thoma in [17, Theorem 3.2]:

Theorem 2.1 Let $G$ a graph and $w$ an even closed walk of $G$. The walk $w$ is primitive if and only if

(i) Every block of $\mathbf{w}$ is a cycle or a cut edge,

(ii) Every multiple edge of the walk $w$ is a double edge of the walk and a cut edge of $\mathbf{w}$,

(iii) Every cut vertex of $\mathbf{w}$ belongs to exactly two blocks and it is a sink of both.

The following corollary was given by E. Reyes, Ch. Tatakis, and A. Thoma in [17, Corollary 3.3] and it describes the underlying graph of a primitive walk, see Fig. 2.

Corollary 2.2 Let $G$ be a graph and let $W$ be a connected subgraph of $G$. The subgraph $W$ is the graph $\mathbf{w}$ of a primitive walk $w$ if and only if 
Fig. 2 A primitive walk

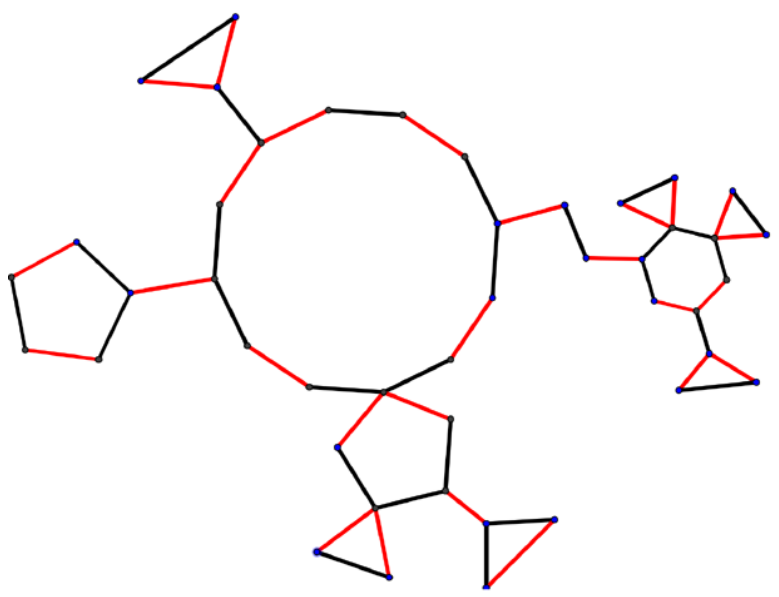

(i) $W$ is an even cycle or

(ii) $W$ is not biconnected and

(a) Every block of $W$ is a cycle or a cut edge and

(b) Every cut vertex of $W$ belongs to exactly two blocks and separates the graph in two parts, the total number of edges of the cyclic blocks in each part is odd.

In this case, the walk $w$ passes through every edge of the cyclic blocks exactly once and from the cut edges twice.

A walk $w$ is primitive if and only if the binomial $B_{w}$ is primitive. The set of primitive binomials form the Graver basis of the toric ideal $I_{G}$. The Graver basis is important to us because every element of a minimal generating set of $I_{G}$ belongs to the Graver basis of $I_{G}$, see [22]. We call a strongly primitive walk a primitive walk that does not have two sinks with distance one in any cyclic block, or equivalently, that does not have two adjacent cut vertices in any cyclic block. For example, the walk in Fig. 1 is primitive but it is not strongly primitive, look, for example, at the cycle with six edges. We say that a binomial is a minimal binomial if it belongs to at least one minimal system of generators of $I_{G}$.

Let $H$ be a subset of $V(G)$ and $G_{H}$ be the induced graph of $H$ in $G$, which is the graph with vertices the elements of the set $H$ and edges the set of edges of $G$ where both vertices belong to $H$. For a given subgraph $F$ of $G$, an edge $f$ of the graph $G$ is called a chord of the subgraph $F$ if the vertices of the edge $f$ belong to $V(F)$ and $f \notin E(F)$. In other words, an edge is called a chord of the subgraph $F$ if it belongs to $E\left(G_{V(F)}\right)$ but not in $E(F)$. A subgraph $F$ is called chordless if $F=G_{V(F)}$. For convenience, by $G_{w}$ we denote the induced graph $G_{V(\mathbf{w})}$, where $w$ is an even closed walk.

Let $w$ be an even closed walk $\left(\left\{v_{1}, v_{2}\right\},\left\{v_{2}, v_{3}\right\}, \ldots,\left\{v_{2 q}, v_{1}\right\}\right)$ and $f=\left\{v_{i}, v_{j}\right\}$ a chord of $w$. Then $f$ breaks $w$ into two walks:

$$
w_{1}=\left(e_{1}, \ldots, e_{i-1}, f, e_{j}, \ldots, e_{2 q}\right)
$$


and

$$
w_{2}=\left(e_{i}, \ldots, e_{j-1}, f\right),
$$

where $e_{s}=\left\{v_{s}, v_{s+1}\right\}, 1 \leq s \leq 2 q$ and $e_{2 q}=\left\{v_{2 q}, v_{1}\right\}$. The two walks are both even or both odd. A chord $e=\left\{v_{k}, v_{l}\right\}$ is called a bridge of a primitive walk $w$ if there exist two different blocks $\mathcal{B}_{1}, \mathcal{B}_{2}$ of $\mathbf{w}$ such that $v_{k} \in \mathcal{B}_{1}$ and $v_{l} \in \mathcal{B}_{2}$. A chord is called even (respectively, odd) if it is not a bridge and breaks the walk in two even walks (respectively, odd). Thus we partition the set of chords of a primitive even walk in three parts: bridges, even chords, and odd chords.

Definition 2.3 Let $w=\left(\left\{v_{i_{1}}, v_{i_{2}}\right\},\left\{v_{i_{2}}, v_{i_{3}}\right\}, \ldots,\left\{v_{i_{2 q}}, v_{i_{1}}\right\}\right)$ be a primitive walk. Let $f=\left\{v_{i_{s}}, v_{i_{j}}\right\}$ and $f^{\prime}=\left\{v_{i_{s^{\prime}}}, v_{i_{j^{\prime}}}\right\}$ be two odd chords (that means not bridges and $j-s, j^{\prime}-s^{\prime}$ are even) with $1 \leq s<j \leq 2 q$ and $1 \leq s^{\prime}<j^{\prime} \leq 2 q$. We say that $f$ and $f^{\prime}$ cross effectively in $w$ if $s^{\prime}-s$ is odd (then necessarily $j-s^{\prime}, j^{\prime}-j, j^{\prime}-s$ are odd) and either $s<s^{\prime}<j<j^{\prime}$ or $s^{\prime}<s<j^{\prime}<j$.

Definition 2.4 We call an $F_{4}$ of the walk $w$ a cycle $\left(e, f, e^{\prime}, f^{\prime}\right)$ of length four which consists of two edges $e, e^{\prime}$ of the walk $w$ both odd or both even, and two odd chords $f$ and $f^{\prime}$ which cross effectively in $w$.

Definition 2.5 Let $w$ be a primitive walk and $f, f^{\prime}$ be two odd chords. We say that $f, f^{\prime}$ cross strongly effectively in $w$ if they cross effectively and they do not form an $F_{4}$ in $w$.

An $F_{4},\left(e_{1}, f_{1}, e_{2}, f_{2}\right)$, separates the vertices of $\mathbf{w}$ into two parts, $V\left(\mathbf{w}_{1}\right), V\left(\mathbf{w}_{2}\right)$, since both edges $e_{1}, e_{2}$ of the $F_{4}$ belong to the same block of $w=\left(w_{1}, e_{1}, w_{2}, e_{2}\right)$.

Definition 2.6 We say that an odd chord $f$ of a primitive walk $w=\left(w_{1}, e_{1}, w_{2}, e_{2}\right)$ crosses an $F_{4},\left(e_{1}, f_{1}, e_{2}, f_{2}\right)$, if one of the vertices of $f$ is in $V\left(\mathbf{w}_{1}\right)$, the other in $V\left(\mathbf{w}_{2}\right)$, and $f$ is different from $f_{1}, f_{2}$.

The next theorem by E. Reyes, Ch. Tatakis, and A. Thoma in [17, Theorem 4.13] gives a necessary and sufficient characterization of minimal binomials of a toric ideal of a graph. This is the main theorem that made the results of this paper possible.

Theorem 2.7 Let $w$ be an even closed walk. $B_{w}$ is a minimal binomial if and only if

(i) $w$ is strongly primitive,

(ii) All the chords of $w$ are odd and there are not two of them which cross strongly effectively and

(iii) No odd chord crosses an $F_{4}$ of the walk $w$.

The support of a monomial $x^{\mathbf{u}}$ of $K\left[x_{1}, \ldots, x_{m}\right]$ is $\operatorname{supp}\left(x^{\mathbf{u}}\right):=\left\{i \mid x_{i}\right.$ divides $\left.x^{\mathbf{u}}\right\}$ and the support of a binomial $B=x^{\mathbf{u}}-x^{\mathbf{v}}$ is $\operatorname{supp}(B):=\operatorname{supp}\left(x^{\mathbf{u}}\right) \cup \operatorname{supp}\left(x^{\mathbf{v}}\right)$. An irreducible binomial $B$ belonging to $I_{A}$ is called a circuit of $I_{A}$ if there is no binomial $B^{\prime} \in I_{A}$ such that $\operatorname{supp}\left(B^{\prime}\right) \varsubsetneqq \operatorname{supp}(B)$. A necessary and sufficient characterization of circuits for toric ideals of graphs was given by R. Villarreal in [23, Proposition 4.2]: 
Theorem 2.8 Let $G$ be a graph. The binomial $B \in I_{G}$ is circuit if and only if $B=B_{w}$, where $w$ is:

(i) An even cycle, or

(ii) Two odd cycles intersecting in exactly one vertex, or

(iii) Two vertex disjoint odd cycles joined by a path.

\section{Complete intersection graphs}

The graph $G$ is called bipartite if it does not contain an odd cycle. The height of $I_{G}$ is equal to $h=m-n+1$ if $G$ is a bipartite graph or $h=m-n$ if $G$ is a non-bipartite graph, where $G$ is a connected graph, $m$ is the number of edges of $G$, and $n$ is the number of its vertices, see [23]. The toric ideal of $G$ is called a complete intersection if it can be generated by $h$ binomials. We say that a graph $G$ is a complete intersection if the ideal $I_{G}$ is a complete intersection.

The problem of determining complete intersection toric ideals has a long history starting with J. Herzog in 1970 [10] and was finally solved by K. Fisher, W. Morris, and J. Shapiro in 1997 [7]. For the history of this problem, see the introduction of [14].

The next theorem says that the complete intersection property of a graph is a hereditary property, in the sense that it holds also for all induced subgraphs.

Theorem 3.1 The graph $G$ is a complete intersection if and only if the graph $G_{H}$ is a complete intersection for every $H \subset V(G)$.

Proof Let $B_{w_{1}}, \ldots, B_{w_{s}}$ be a minimal system of generators of $I_{G_{H}}$, for some even closed walks $w_{i}$ of $G, 1 \leq i \leq s$. A minimal generator $B_{w}$ of $I_{G_{H}}$ is always a minimal generator of $I_{G}$ since the property of being minimal generator depends only on the induced graph $G_{w}$ of $w$, see Theorem 2.7. Note that for a walk $w$ of $G_{H}$, the induced graph $G_{w}$ is the same in $G_{H}$ as in $G$. Therefore, we can extend $B_{w_{1}}, \ldots, B_{w_{s}}$ to a minimal system of generators $B_{w_{1}}, B_{w_{2}}, \ldots, B_{w_{h}}$ of $I_{G}, s \leq h$. The toric ideal $I_{G}$ is complete intersection, therefore $B_{w_{1}}, \ldots, B_{w_{h}}$ is a regular sequence. Since the ideal $I_{G}$ in $K\left[e_{1}, \ldots, e_{m}\right]$ is homogeneous and none of the variables is a zero divisor in the edge ring $K[G]=K\left[e_{1}, \ldots, e_{m}\right] / I_{G}$, the sequence $B_{w_{1}}, \ldots, B_{w_{s}}$ is regular, and therefore $I_{G_{H}}$ is a complete intersection toric ideal, see [18].

The converse is obvious since for $H=V(G)$ we have $G=G_{H}$.

The next proposition gives a very useful property of complete intersection toric ideals that will play a crucial role in the proofs of the theorems in the next sections.

Proposition 3.2 If $G$ is a complete intersection and $B_{w_{1}}, \ldots, B_{w_{s}}$ is a minimal set of generators of the ideal $I_{G}$ then there are no two walks $w_{i}, w_{j}, i \neq j$ such that $\mathbf{w}_{i}^{+} \cap \mathbf{w}_{j}^{+} \neq \emptyset$ and $\mathbf{w}_{i}^{-} \cap \mathbf{w}_{j}^{-} \neq \emptyset$, or $\mathbf{w}_{i}^{+} \cap \mathbf{w}_{j}^{-} \neq \emptyset$ and $\mathbf{w}_{i}^{-} \cap \mathbf{w}_{j}^{+} \neq \emptyset$.

Proof Let $B_{w_{1}}=\mathbf{e}^{w_{1}^{+}}-\mathbf{e}^{w_{1}^{-}}, \ldots, B_{w_{s}}=\mathbf{e}^{w_{s}^{+}}-\mathbf{e}^{w_{s}^{-}}$be a minimal set of generators of the complete intersection toric ideal $I_{G}$. Then the matrix $M$ with rows $w_{i}^{+}-w_{i}^{-}$ 
is mixed dominating, see Corollary 2.10 [8]. A matrix is called mixed if its every row contains both a positive and a negative entry and dominating if it does not contain a square mixed submatrix. Suppose that there exist $B_{w_{1}}, \ldots, B_{w_{s}}$, a minimal set of generators, and two walks $w_{i}, w_{j}, i \neq j$ such that $\mathbf{w}_{i}^{+} \cap \mathbf{w}_{j}^{+} \neq \emptyset$ and $\mathbf{w}_{i}^{-} \cap \mathbf{w}_{j}^{-} \neq \emptyset$. Let $e_{k} \in \mathbf{w}_{i}^{+} \cap \mathbf{w}_{j}^{+}$and $e_{l} \in \mathbf{w}_{i}^{-} \cap \mathbf{w}_{j}^{-}$. Then the $2 \times 2$ square submatrix taken from the $i, j$ rows and $k, l$ columns is mixed, contradicting the fact that $M$ is dominating. The proof of the other part is similar.

It follows from Proposition 3.2 that if two edges are consecutive edges in two even closed walks $w_{1}$ and $w_{2}$ in a complete intersection graph then both $B_{w_{1}}, B_{w_{2}}$ cannot belong to the same minimal system of generators of $I_{G}$. Also note that you cannot have in a minimal system of generators two circuits with two odd cycles and one of the cycles being the same in both, since any cycle contains at least three edges, and therefore there are at least two consecutive edges in common. For toric ideals of graphs, Theorem 2.7 determines the form of a minimal binomial. Two minimal binomials sometimes belong to a minimal system of generators of the toric ideal, but for certain minimal binomials it is impossible to find a minimal system of generators that contains both of them, see [3]. For a toric ideal $I_{A}$, if two minimal binomials have different $A$-degrees then there exists a minimal system of generators for $I_{A}$ that contains both of them. But if they have the same $A$-degree, sometimes there exists a minimal system of generators for $I_{A}$ that contains both of them and sometimes not; for more details, look at [3]. For toric ideals of graphs, the situation is simpler. Let $B_{w}, B_{w^{\prime}}$ be two minimal generators of $I_{G}$. Then there exist a minimal system of generators for $I_{G}$ that contains both of them if and only if $w$ and $w^{\prime}$ are not $F_{4}$ equivalent, see [17].

Definition 3.3 Two primitive walks $w, w^{\prime}$ differ by an $F_{4}, \xi=\left(e_{1}, f_{1}, e_{2}, f_{2}\right)$, if $w=\left(w_{1}, e_{1}, w_{2}, e_{2}\right)$ and $w^{\prime}=\left(w_{1}, f_{1},-w_{2}, f_{2}\right)$, where both $w_{1}, w_{2}$ are odd walks. Two primitive walks $w, w^{\prime}$ are $F_{4}$-equivalent if either $w=w^{\prime}$ or there exists a series of walks $w_{1}=w, w_{2}, \ldots, w_{n-1}, w_{n}=w^{\prime}$ such that $w_{i}$ and $w_{i+1}$ differ by an $F_{4}$, where $1 \leq i \leq n-1$.

For more information about minimal system of generators of toric ideals of graphs, see [17].

\section{On the blocks of a complete intersection graph}

Theorem 4.2 is one of the main results of the article and proves that if a complete intersection graph has $n$ blocks then at least $n-2$ of them are bipartite. In the case that there are two non-bipartite blocks, they have to have a special position in the graph, namely, they have to be contiguous.

Definition 4.1 Two blocks of a graph $G$ are called contiguous if they have a common cut vertex or there is a path from the one to the other in which each edge of the path belongs to a different block. 
Let $B(G)$ be the block tree of $G$, the bipartite graph with bipartition $(\mathbb{B}, \mathbb{S})$ where $\mathbb{B}$ is the set of blocks of $G$ and $\mathbb{S}$ is the set of cut vertices of $G,\{\mathcal{B}, v\}$ is an edge if and only if $v \in \mathcal{B}$. The leaves of the block tree are always blocks and are called end blocks. Let $\mathcal{B}_{k}, \mathcal{B}_{i}, \mathcal{B}_{l}$ be blocks of a graph $G$. We call the block $\mathcal{B}_{i}$ an internal block of $\mathcal{B}_{k}, \mathcal{B}_{l}$, if $\mathcal{B}_{i}$ is an internal vertex in the unique path defined by $\mathcal{B}_{k}, \mathcal{B}_{l}$ in the tree $B(G)$. Every path from $\mathcal{B}_{k}$ to $\mathcal{B}_{l}$ passes from every internal block of $\mathcal{B}_{k}, \mathcal{B}_{l}$ and has as vertices at least the cut vertices which are vertices in the path $\left(\mathcal{B}_{k}, \ldots, \mathcal{B}_{l}\right)$ in $B(G)$. In the case that $\mathcal{B}_{i}$ is an internal block of $\mathcal{B}_{k}, \mathcal{B}_{l}$ we denote by $y_{i, k}$ and $y_{i, l}$ the cut vertices of $\mathcal{B}_{i}$ which are also vertices of the unique path $\left(\mathcal{B}_{k}, \ldots, \mathcal{B}_{l}\right)$ in the blocktree $B(G)$. It follows from the definition and the above remarks that if the blocks $\mathcal{B}_{k}$, $\mathcal{B}_{l}$ are contiguous then $\left\{y_{i, k}, y_{i, l}\right\}$ is an edge of the graph $G$. Even more, there is a unique chordless path between two contiguous blocks $\mathcal{B}_{k}, \mathcal{B}_{l}$, where every edge is in the form $\left\{y_{i, k}, y_{i, l}\right\}$.

\section{Theorem 4.2 Let $G$ be a graph. If $G$ is complete intersection then either}

(i) All blocks of $G$ are bipartite, or

(ii) All blocks are bipartite except one, or

(iii) All blocks are bipartite except two which are contiguous.

Proof Let $G$ be a complete intersection graph and let $\mathcal{B}_{1}, \ldots, \mathcal{B}_{t}$ be its blocks. We assume that $G$ has three or more non-bipartite blocks and let three of them be $\mathcal{B}_{m}, \mathcal{B}_{k}, \mathcal{B}_{l}$. At least one of $\mathcal{B}_{m}, \mathcal{B}_{k}, \mathcal{B}_{l}$ is not an internal block of the other two, let it be $\mathcal{B}_{m}$. We denote by $y_{i, j}$ the cut vertex of $\mathcal{B}_{i}$ which is the second vertex of the unique path $\left(\mathcal{B}_{i}, \ldots, \mathcal{B}_{j}\right)$ in the block tree $B(G)$, where $i, j \in\{m, k, l\}$. The block $\mathcal{B}_{i}$, $i \in\{m, k, l\}$, is non-bipartite, therefore it contains at least one odd cycle, but it is biconnected, therefore through every point of it passes an odd cycle. We denote by $c_{i, j}$ an odd cycle of the block $\mathcal{B}_{i}$ which contains the vertex $y_{i, j}$ and with the smallest number of edges. Let $w_{m, k}=\left(c_{m, k}, p_{m, k}, c_{k, m},-p_{m, k}\right), w_{m, l}=\left(c_{m, l}, p_{m, l}, c_{l, m},-p_{m, l}\right)$, where $p_{m, k}, p_{m, l}$ are chordless paths from $y_{m, k}$ to $y_{k, m}$ and from $y_{m, l}$ to $y_{l, m}$, respectively, and we can choose $c_{m, k}=c_{m, l}$ since $\mathcal{B}_{m}$ is not an internal block of the other two. Note that whenever there is a path from a vertex to another one then there is a chordless path between these two vertices.

We claim that the binomials $B_{w_{m, k}}$ and $B_{w_{m, l}}$ are minimal. First, $B_{w_{m, k}}$ is a circuit, see Theorem 2.8, and therefore $w_{m, k}$ is primitive, actually strongly primitive, see Theorem 2.7. Note that $w_{m, k}$ has no bridges, since bridges are chords of the walk $w_{m, k}$ that have their vertices in different blocks of $\mathbf{w}_{m, k}$ which is impossible since (a) $p_{m, k}$ is chordless, thus there is no bridge from the blocks of the path to themselves, (b) $y_{m, k}, y_{k, m}$ are cut vertices, thus there is no bridge from the cycles to the path, and (c) the odd cycles are of minimum length, therefore there is no chord of the cycles incident to $y_{m, k}$ or $y_{k, m}$. Also $w_{m, k}$ has no even chords since $c_{m, k}, c_{k, m}$ are odd cycles of minimum length. So all the chords of $w_{m, k}$ are odd. Note that the odd chords of $w_{m, k}$ are chords of either the cycle $c_{m, k}$ or $c_{k, m}$. There are no two of them which cross effectively, except if they form an $F_{4}$, otherwise there will be an other odd cycle with strictly smaller number of edges than either $c_{m, k}$ or $c_{k, m}$ which passes from $y_{m, k}$ or $y_{k, m}$. Also no odd chord crosses an $F_{4}$ of $w_{m, k}$, since an odd chord that crosses an $F_{4}$ of $w_{m, k}$ is an even chord for an $F_{4}$-equivalent walk of $w_{m, k}$, which contradicts 
the minimallity of one of the cycles $c_{m, k}$ or $c_{k, m}$. Therefore, by Theorem $2.7, B_{w_{m, k}}$ is minimal. Similarly for the binomial $B_{w_{m, l}}$. Note that $\operatorname{deg}_{G}\left(B_{w_{m, k}}\right) \neq \operatorname{deg}_{G}\left(B_{w_{m, l}}\right)$ thus they may belong to the same minimal system of generators of $I_{G}$. This contradicts Proposition 3.2 since the cycle $c_{m, k}=c_{m, l}$ is contained in both walks. So the graph $G$ has at most two non-bipartite blocks.

Suppose that we are in the case that $G$ has exactly two non-bipartite blocks and let them be $\mathcal{B}_{1}$ and $\mathcal{B}_{2}$. We will prove that the blocks $\mathcal{B}_{1}$ and $\mathcal{B}_{2}$ are contiguous. Suppose not, then there exists at least one block $\mathcal{B}_{t}$ such that every path from $\mathcal{B}_{1}$ to $\mathcal{B}_{2}$ has at least two edges in $\mathcal{B}_{t}$, see the remarks after the definition of contiguity. Let $y_{t, 1}$ and $y_{t, 2}$ be the cut vertices of $\mathcal{B}_{t}$ which are also vertices of the unique path $\left(\mathcal{B}_{1}, \ldots, \mathcal{B}_{2}\right)$ in the block-tree $B(G)$. Since $y_{t, 1}$ and $y_{t, 2}$ belong to the same block $\mathcal{B}_{t}$, there exist at least two internally disjoint paths of length at least two connecting them. Note that $\left\{y_{t, 1}, y_{t, 2}\right\}$ is not an edge of $G$, thus there are two different chordless paths from $y_{t, 1}$ to $y_{t, 2}$. Thus there exist at least two different chordless paths from $y_{1,2}$ and $y_{2,1}$. Therefore, by choosing the odd cycles $c_{1,2}$ and $c_{2,1}$ as in the above construction, and the two chordless paths $p_{1}, p_{2}$ from $y_{1,2}$ and $y_{2,1}$ we get two even walks $w_{1}=\left(c_{1,2}, p_{1}, c_{2,1},-p_{1}\right), w_{2}=\left(c_{1,2}, p_{2}, c_{2,1},-p_{2}\right)$. As before, there are no bridges in both $w_{1}, w_{2}$ and since all the chords of $c_{1,2}$ and $c_{2,1}$ are odd, there are no two of them which cross effectively (except if they form an $F_{4}$ ) and no odd chord crosses an $F_{4}$ of $w_{m, k}$, each one of those paths will give a minimal generator of $I_{G}$. Note that $\operatorname{deg}_{G}\left(B_{w_{1}}\right) \neq \operatorname{deg}_{G}\left(B_{w_{2}}\right)$ thus they may belong to the same minimal system of generators of $I_{G}$. This contradicts Proposition 3.2 since the cycles $c_{1,2}$ and $c_{2,1}$ are contained in both walks. So $\mathcal{B}_{1}$ and $\mathcal{B}_{2}$ are contiguous blocks.

\section{Circuit ideals and complete intersections}

The first theorem of this section states an interesting property of toric ideals of graphs: Complete intersection toric ideals of graphs are circuit ideals. Note that complete intersection toric ideals usually do not have this property. For more information on circuit ideals, see the article [2] by T. Bogart, A.N. Jensen, and R.R. Thomas; and for toric ideals of homogeneous normal configurations generated by circuits, see the article [13] by J. Martinez-Bernal and R.H. Villarreal. The configurations of vectors arising from graphs are always homogeneous but not necessarily normal, even if the graphs are complete intersections. Thus these results cannot be applied in the general case of graphs. In the next section, we study normality and we determine all complete intersection graphs for which their corresponding configurations of vectors are normal, i.e., $\mathbb{N} A=\mathbb{Z} A \cap \mathbb{R}_{+} A$.

Theorem 5.1 Let $G$ be a graph. If $G$ is a complete intersection then every minimal generator of $I_{G}$ is a circuit.

Proof Suppose that $I_{G}$ is a complete intersection toric ideal that has a minimal generator $B_{w}$ which is not a circuit. Since the binomial $B_{w}$ is minimal it is also primitive. Therefore, all of the blocks of $\mathbf{w}$ are cycles or cut edges, see Theorem 2.7. The end blocks of $\mathbf{w}$ are always cyclic blocks and if there is more than one end block they are 
all odd cycles, see Corollary 2.2. Since $B_{w}$ is not a circuit it has at least three cyclic blocks from which at least two are end blocks and therefore odd, see Theorems 2.7 and 2.8, otherwise, if it has only one cyclic block then there is only one end block in $\mathbf{w}$ and therefore $\mathbf{w}$ is an even cycle, and if it has exactly two cyclic blocks, they should be end blocks and the cut edges should be internal blocks, thus in all cases $\mathbf{w}$ is a circuit, see Theorem 2.8 .

The graph $G$ is a complete intersection; therefore, the induced graph $G_{w}$ is a complete intersection, by Theorem 3.1, where $G_{w}$ is the induced graph of $\mathbf{w}$ in $G$. Note that the walk $w$ has no bridges, since $B_{w}$ is a minimal generator, see Theorem 2.7; therefore, there is a one-to-one correspondence between the blocks of $\mathbf{w}$ and the blocks of $G_{w}$. Cut edges of $\mathbf{w}$ are cut edges of $G_{w}$, but cyclic blocks of $\mathbf{w}$ may have chords in $G_{w}$. At least two of the blocks are non-bipartite since they have an odd cycle. Therefore, by Theorem 4.2, there are exactly two. There are at least two end blocks of $\mathbf{w}$, and thus they are odd cycles. Therefore, the two non-bipartite blocks are the only end blocks of the block graph of $G_{w}$, which means that the block tree $B\left(G_{w}\right)$ is a path, see Corollary 2.2. Let $\mathcal{B}_{1}, \mathcal{B}_{2}$ be the two odd cyclic blocks of $\mathbf{w}$ and $\mathcal{B}_{3}$ be one of the other cyclic blocks, then $\mathcal{B}_{3}$ will be an internal block of $\mathcal{B}_{1}, \mathcal{B}_{2}$. From Theorem 4.2, the two blocks $\mathcal{B}_{1}, \mathcal{B}_{2}$ are contiguous; therefore, there will be an edge of the block $\mathcal{B}_{3}$ at the path which connects the two odd cycles $\mathcal{B}_{1}, \mathcal{B}_{2}$ of $w$. If the edge belongs to the walk $w$ then $w$ is not strongly primitive, and if the edge does not belong to $w$ then it is a bridge of $w$ since its vertices are cut vertices of $\mathbf{w}$ and thus belong to two different blocks of $\mathbf{w}$. In both cases, Theorem 2.7 implies that $B_{w}$ is not a minimal generator, a contradiction. Therefore, $\mathbf{w}$ has at most two cyclic blocks and thus $B_{w}$ is a circuit, see Theorem 2.1 and Theorem 2.8.

The next proposition will be useful in the proof of Theorem 5.3.

Proposition 5.2 Let $G$ be a complete intersection graph and let $B_{w}$ be a minimal generator of $I_{G}$. If $w$ is not an even cycle then $w$ is chordless.

Proof From Theorem 5.1, the walk $w$ consists of two odd edge-disjoint cycles joint at vertex or with a path, see Theorem 2.8. Thus $w$ is of the form $\left(c_{1}, p, c_{2},-p\right)$, where $c_{1}, c_{2}$ are odd cycles $y_{1}, y_{2}$ are points of $c_{1}$ and $c_{2}$, respectively, and $p$ is a path from $y_{1}$ to $y_{2}$; and it is possible that $y_{1}=y_{2}$ and $p$ is empty. Since the binomial $B_{w}$ is minimal, the walk $w$ has no even chords and no bridges, see Theorem 2.7. Suppose that the walk $w$ had an odd chord $e=\{a, b\}$, then from the definition of an odd chord, both vertices belong to the same cycle. Without loss of generality, we can suppose that both vertices $a, b$ belong to the cycle $c_{1}$. Then $c_{1}=\left(c_{11}, c_{12}, c_{13}\right)$, where $c_{11}, c_{12}, c_{13}$ are nonempty paths from $y_{1}$ to $a, a$ to $b$ and $b$ to $y_{1}$, respectively. Among all possible such odd chords $e$, we choose the vertex $a$ in such a way that the length of $c_{11}$ is as small as possible. If there are more than one odd chord with one vertex $a$ then we choose $b$ such that $c_{12}$ is as small as possible. By the choice of $a$, the walk $w_{1}=\left(c_{12},\{b, a\},-c_{11}, p, c_{2},-p, c_{11}\right)$ has no bridge from $c_{12}$ to $c_{11}$. By the choice of $b$, there is no bridge from $c_{12}$ to the vertex $a$. Note that for $w_{1}$ it is not possible to have another bridge since $w$ has no bridges. Any chord of the odd cycle $\left(c_{12}, e\right)$ is also a chord of $w . B_{w}$ is minimal generator of $I_{G}$; therefore, if there 
exist such chords then all of them are odd chords of $w$ and no two of them cross strongly effectively and no odd chord crosses an $F_{4}$ of the walk $w$, see Theorem 2.7. Therefore, also $B_{w_{1}}$ is minimal. Note that $\operatorname{deg}_{G}\left(B_{w}\right) \neq \operatorname{deg}_{G}\left(B_{w_{1}}\right)$ thus they may belong to the same minimal system of generators of $I_{G}$. This is a contradiction to Proposition 3.2 since the cycle $c_{2}$ is contained in both walks. Therefore, $w$ has no chord.

Theorem 5.3 Let $G$ be a biconnected complete intersection graph $G$. All minimal generators of $I_{G}$ are in the form $B_{w}$ where $w$ is an even cycle.

Proof Theorem 5.1 implies that all generators are circuits, thus to prove the theorem we will suppose that there is an even closed walk $w=\left(c_{1}, p, c_{2},-p\right)$ of $G$ such that $B_{w}$ is a minimal generator of $I_{G}$ and we will arrive to a contradiction, where $c_{1}, c_{2}$ are odd cycles of $G, p=\left(v_{1}, \ldots, v_{l}\right)$ a path between them denoted by its vertices, $V\left(c_{1}\right) \cap V(p)=\left\{v_{1}\right\}, V\left(c_{2}\right) \cap V(p)=\left\{v_{l}\right\}$, and $V\left(c_{1}\right) \cap V\left(c_{2}\right)=\emptyset$, except in the case that the path $p$ is just a vertex, $\left\{v_{1}\right\}$, and then $V\left(c_{1}\right) \cap V\left(c_{2}\right)=\left\{v_{1}\right\}$.

We claim that there is a path from $c_{1}$ to $c_{2}$ which is vertex disjoint from $p$. Suppose not. Then, since $v_{1}$ is not a cut vertex of $G$ there must be at least one $H$ path from $V\left(c_{1}\right)-\left\{v_{1}\right\}$ to $\left\{v_{2}, \ldots, v_{l}\right\}$, where $H$ is the graph $\mathbf{c}_{1} \cup \mathbf{c}_{2} \cup \mathbf{p}$. Consider among all such $H$-paths those that for their end point $v_{k}$ the $k$ is maximum, $2 \leq k \leq l$. Among those let $\zeta$ be one of minimal length. Let $z$ be the even closed walk $\left(c_{1}, \zeta,\left\{v_{k}, v_{k+1}\right\}, \ldots,\left\{v_{l-1}, v_{l}\right\}, c_{2},\left\{v_{l}, v_{l-1}\right\}, \ldots\left\{v_{k+1}, v_{k}\right\},-\zeta\right)$. Then $\operatorname{deg}_{G}\left(B_{z}\right) \neq \operatorname{deg}_{G}\left(B_{w}\right)$, except in the case that $\zeta$ consists of only one edge and $k=2$. But then this edge is a bridge of the walk $w$, contradicting the minimality of $B_{w}$, see Theorem 2.7. Then the binomial $B_{z}$ cannot be a minimal generator since $w$ and $z$ share the cycle $c_{2}$. Therefore, $\mathbf{z}$ must have at least a chord. But $w$ is chordless, see Proposition 5.2. There is no chord from $c_{2}$ to $\zeta$, otherwise there is a path from $c_{1}$ to $c_{2}$ which is vertex disjoint from $p$. There is no chord from $\left\{v_{k}, v_{k+1} \ldots, v_{l-1}, v_{l}\right\}$ to $\zeta$ since the existence of a chord like that contradicts the choice of $k$. The only choice left for a chord is from the cycle $c_{1}$ to $\zeta$. And since $\zeta$ is a path of minimal length any chord should be from $c_{1}$ to the second vertex of the path $\zeta$, say $x$. Look at the induced graph of $\mathbf{c}_{1} \cup\{x\}$. Since $c_{1}$ is chordless any chord of $\mathbf{c}_{1} \cup\{x\}$ is from $c_{1}$ to $x$. There must be at least two chords of $\mathbf{c}_{1} \cup\{x\}$, since also the first edge of $\zeta$ is a chord of $\mathbf{c}_{1} \cup\{x\}$. Look at the chordless cycles passing through $x$, in the induced graph of $\mathbf{c}_{1} \cup\{x\}$. At least one of the cycles is odd since $c_{1}$ is odd and each one consists of edges of $c_{1}$ and two chords passing through $x$. Let $o$ be an odd such cycle, then the even closed walk $z^{\prime}=\left(o_{1}, \zeta^{\prime},\left\{v_{k}, v_{k+1}\right\}, \ldots,\left\{v_{l-1}, v_{l}\right\}, c_{2},\left\{v_{l}, v_{l-1}\right\}, \ldots\left\{v_{k+1}, v_{k}\right\},-\zeta^{\prime}\right)$ has no chords and bridges, where $\zeta^{\prime}$ is the subpath of $\zeta$ from $x$ to $v_{k}$. Therefore, by Theorem 2.7, $B_{z^{\prime}}$ is a minimal generator of $I_{G}$. But this is not possible since $\operatorname{deg}_{G}\left(B_{w}\right) \neq$ $\operatorname{deg}_{G}\left(B_{z^{\prime}}\right)$ and $z^{\prime}$ has a common cycle, the $c_{2}$, with $w$.

Therefore, there is a path from $c_{1}$ to $c_{2}$ which is vertex disjoint from $p$. Let $q=$ $\left(x_{1}, y_{1}, \ldots, y_{2}, x_{2}\right)$ be one of minimal length, where the vertex $x_{1} \in c_{1}$ and the vertex $x_{2} \in c_{2}$. Note that the length of $q$ is greater than one, since otherwise it will be just an edge which will be a bridge of $w$ and then $B_{w}$ will not be a minimal generator of $I_{G}$. Note also that it may be $y_{1}=y_{2}$. Look at the graph induced by the graph $\mathbf{w} \cup \mathbf{q}$. By Proposition 5.2, $w$ has no chords and the path $q$ has minimal length. Therefore, the 
chords of $\mathbf{w} \cup \mathbf{q}$ are either edges from the cycle $c_{1}$ to $y_{1}$, or from the cycle $c_{2}$ to $y_{2}$ and from $p$ to $q$ except to the vertices $x_{1}, x_{2}$.

We claim that there are chords from the cycle $c_{1}$ to $y_{1}$. Suppose not. Let $c_{11}$ be the path of greater length from $v_{1}$ to $x_{1}$ on the cycle $c_{1}$ and $c_{12}$ be the path of smaller length from $v_{1}$ to $x_{1}$ on the circle $c_{1}$. Note that the cycle $c_{1}$ is odd and also the length of $c_{11}$ is greater than one. Denote by $c_{21}$ the path from $x_{2}$ to $v_{2}$ such that the cycle $w^{\prime}=\left(c_{11}, q, c_{21},-p\right)$ is even. There exists such a path since the cycle $c_{2}$ is odd. Consider $c$ to be the smallest even cycle of the form $\left(c_{11}, c^{\prime}\right)$ and the edges of it being edges or chords of $w^{\prime}$. Note that there exists such a cycle since $w^{\prime}$ is of that form. Note that $c$ is a cycle; therefore, it has no bridges and it is strongly primitive. Also from the minimality of the length of $c$ among even cycles of the form $\left(c_{11}, c^{\prime}\right)$, $c$ has no even chord and no two odd chords which cross strongly effectively and no odd chord that crosses an $F_{4}$ of $c$. Otherwise the proofs of Propositions 4.8 and 4.12 of [17] show that there exist two smaller even cycles and one of them is of the form $\left(c_{11}, c^{\prime \prime}\right)$ since there are no chords from $c_{11}$. Then $B_{c}$ is a minimal generator of $I_{G}$. Note that $\operatorname{deg}_{G}\left(B_{w}\right) \neq \operatorname{deg}_{G}\left(B_{c}\right)$ thus they may belong to the same minimal system of generators of $I_{G}$. This contradicts Proposition 3.2 since the edges of $c_{11}$ are contained in both walks and the length of $c_{11}$ is greater than one.

Therefore, there exist chords from the cycle $c_{1}$ to $y_{1}$ and similarly from the cycle $c_{2}$ to $y_{2}$. Let $\left(u_{1,1}, u_{1,2}, \ldots, u_{1, s_{1}}\right)$ be the cycle $c_{1}$ denoted by its vertices, where $u_{1,1}=v_{1}$. We consider all chords from the cycle $c_{1}$ to $y_{1}$ together with the edge $\left\{x_{1}, y_{1}\right\}$ and denote them by $e_{1}=\left\{y_{1}, u_{1, i_{1}}\right\}, \ldots, e_{t_{1}}=\left\{y_{1}, u_{1, i_{t_{1}}}\right\}$, where $i_{1}<\cdots<i_{t_{1}}$. Then the cycles $c_{1, j}=\left(y_{1}, u_{1, i_{j}}, u_{1, i_{j}+1}, \ldots, u_{1, i_{j+1}}\right)$, for $1 \leq j \leq$ $t_{1}-1$, and $c_{1, t_{1}}=\left(y_{1}, u_{1, i_{t_{1}}}, u_{1, i_{t_{1}+1}}, \ldots, u_{1, s_{1}}, u_{1,1}, \ldots, u_{1, i_{1}}\right)$ are chordless. If one of them were even then, since it does not have any chords, the corresponding binomial would be a minimal generator of $I_{G}$, see Theorem 2.7. But it cannot have two consecutive edges which are in $\mathbf{w}^{+}$or $\mathbf{w}^{-}$; therefore, the only choice for the cycle is $\left(y_{1}, u_{1, s_{1}}, u_{1,1}, u_{1,2}\right)$.

Similarly, there cannot be two consecutive odd cycles $c_{1, j}, c_{1, j+1}$ since then the cycle $c=\left(y_{1}, u_{1, i_{j}}, u_{1, i_{j}+1}, \ldots, u_{1, i_{j+1}}, u_{1, i_{j+1}+1}, u_{1, i_{j+2}}\right)$ is even with only one odd chord; therefore, the corresponding binomial is a minimal generator, see Theorem 2.7. But $c$ cannot have two consecutive edges which are in $\mathbf{w}^{+}$or $\mathbf{w}^{-}$; therefore, the only choice for the resulting cycle is $\left(y_{1}, u_{1, s_{1}}, u_{1,1}, u_{1,2}\right)$, and the two original cycles where $\left(y_{1}, u_{1,1}, u_{1,2}\right)$ and $\left(y_{1}, u_{1, s_{1}}, u_{1,1}\right)$. Since the cycle $c_{1}$ is odd and each cycle $c_{1, j}$ consists of a part of $c_{1}$ and two new edges, the number of odd cycles $c_{1, j}$ must be odd. And since among these cycles at most one can be even, then the number of odd cycles cannot be greater than or equal to three since then you can find two consecutive cycles different from $\left(y_{1}, u_{1,1}, u_{1,2}\right)$ and $\left(y_{1}, u_{1, s_{1}}, u_{1,1}\right)$. But then there is only one choice left, one cycle is even, the $\left(y_{1}, u_{1, s_{1}}, u_{1,1}, u_{1,2}\right)$, and one odd, the $\left(y_{1}, u_{1,2}, u_{1,3}, \ldots, u_{1, s_{1}}\right)$.

A similar statement is also true for $y_{2}$ and the cycle $c_{2}$. The even closed walk $z=$ $\left(y_{1}, u_{1,2}, u_{1,3}, \ldots, u_{1, s_{1}}, y_{1}, \xi^{\prime}, y_{2}, u_{2,2}, u_{2,3}, \ldots, u_{2, s_{2}}, y_{2},-\xi^{\prime}, y_{1}\right)$ has no chords or bridges; therefore, $B_{z}$ is a minimal generator of $I_{G}$, see Theorem 2.7, where $\xi^{\prime}$ is the subpath of $q$ from $y_{1}$ to $y_{2}$. But then from Proposition 3.2 and that fact that $B_{w}$ is a minimal generator we conclude that $s_{1}=3=s_{2}$.

Look at the graph $\mathbf{z} \cup \mathbf{w}$, the only chords of the graph can be from the path $p$ to the path $\xi^{\prime}$. Let $e=\{a, b\}$ be the nearest chord to the vertex $u_{1,1}$, if there exists 
one, otherwise call $e$ the chord $\left\{a=u_{2,1}, b=u_{2,3}\right\}$. By the choice of the edge $e$, the cycle $o=\left(u_{1,1}, \ldots, a, b, \ldots, y_{1}, u_{1,3}, u_{1,2}\right)$ has only two chords, $\left\{u_{1,2}, y_{1}\right\}$ and $\left\{u_{1,1}, u_{1,3}\right\}$.

There are two cases. The first case: the cycle $o=\left(u_{1,1}, \ldots, a, b, \ldots, y_{1}, u_{1,3}, u_{1,2}\right)$ is even. In this case, the two chords $\left\{u_{1,2}, y_{1}\right\},\left\{u_{1,1}, u_{1,3}\right\}$ form an $F_{4}$ of the even closed walk $o$. Then $B_{o}$ is a minimal generator of $I_{G}$, see Theorem 2.7. But this is impossible since the minimal generators $B_{w}, B_{o}$ have two consecutive edges in common, $\left\{u_{1,3}, u_{1,2}\right\}$ and $\left\{u_{1,2}, u_{1,1}\right\}$, and $\operatorname{deg}_{G}\left(B_{w}\right) \neq \operatorname{deg}_{G}\left(B_{o}\right)$, see Proposition 3.2 .

The second case: the cycle $o$ is odd, then the cycles $o_{1}=\left(u_{1,1}, \ldots, a, b, \ldots, y_{1}\right.$, $\left.u_{1,2}\right), o_{2}=\left(u_{1,1}, \ldots, a, b, \ldots, y_{1}, u_{1,3}\right)$ are both chordless and even. But then the $B_{o_{1}}, B_{O_{2}}$ are minimal generators of $I_{G}$. But this contradicts Proposition 3.2 since the two cycles have all edges in common except for two and $\operatorname{deg}_{G}\left(B_{O_{1}}\right) \neq \operatorname{deg}_{G}\left(B_{O_{2}}\right)$.

We conclude that all minimal generators of $I_{G}$ are of the form $B_{w}$ where $w$ is an even cycle.

Theorem 5.4 Let $G$ be a complete intersection graph. All minimal generators, except for at most one, of $I_{G}$ are of the form $B_{w}$ where $w$ is an even cycle. The possible exceptional generator is a circuit whose two odd cycles belong to two different contiguous blocks.

Proof In the case that all blocks of $G$ are bipartite or all except one, there is no generator of the form $B_{w}$ where $w=\left(c_{1}, p, c_{2},-p\right)$ with $c_{1}, c_{2}$ odd cycles, see Theorem 5.3. In the case that $G$ has two contiguous non-bipartite blocks, say $\mathcal{B}_{1}, \mathcal{B}_{2}$, according to the proof of Theorem 4.2 , there is one generator of the form $B_{w}$, where $w=\left(c_{1}, p, c_{2},-p\right)$ is an even closed walk, where $c_{1}, c_{2}$ are odd chordless cycles of $\mathcal{B}_{1}, \mathcal{B}_{2}$ that are passing through $y_{1,2}$ and $y_{2,1}$, respectively, and $p$ is the unique chordless path between them. Suppose that there is another generator of the form $B_{w^{\prime}}$ where $w^{\prime}$ is not an even cycle. Then from Theorem $5.3, \mathbf{w}^{\prime}$ is not contained in the blocks $\mathcal{B}_{1}$ or $\mathcal{B}_{2}$. So $\mathbf{w}^{\prime}$ consists of an odd chordless cycle $c_{1}^{\prime}$ in the block $\mathcal{B}_{1}$, see Proposition 5.2, an odd chordless cycle $c_{2}^{\prime}$ in the block $\mathcal{B}_{2}$ and a path $\xi$ from the one to the other. The path $\xi$ consists of three paths. A chordless path $p_{1}$ is from the cycle $c_{1}^{\prime}$ to $y_{1,2}$, the second is $p$ (since otherwise the path $\xi$ has a chord which plays the role of a bridge and destroys the minimality of the generator $B_{w^{\prime}}$, see Theorem 2.7), and finally, a chordless path $p_{2}$ is from the cycle $c_{2}^{\prime}$ to $y_{2,1}$. Some of them may be empty. But then for the even closed walk $w^{\prime \prime}=\left(c_{1}^{\prime}, p_{1}, p, c_{2},-p,-p_{1}\right)$ we know that (i) the two odd cycles $c_{1}^{\prime}, c_{2}$ are chordless, (ii) the path $\left(p_{1}, p\right)$ is chordless, (iii) there is no chord from $c_{1}^{\prime}$ to $p$ since $c_{1}^{\prime}$ is in the block $\mathcal{B}_{1}$, (iv) there is no chord from $c_{2}$ to $p$ since $c_{2}$ is in the block $\mathcal{B}_{2}$, and $(\mathrm{v})$ there is no chord from $c_{1}^{\prime}$ to $p_{1}$ since then it will be a bridge of $w^{\prime}$ which is impossible by Theorem 2.7. Combining all these, Theorem 2.7 says that $B_{w^{\prime \prime}}$ is a minimal generator. The walks $w^{\prime \prime}$ and $w$ have more than two consecutive edges in common and $B_{w^{\prime \prime}}$, $B_{w}$ are minimal generators and $\operatorname{deg}_{G}\left(B_{w^{\prime \prime}}\right) \neq \operatorname{deg}_{G}\left(B_{w}\right)$, thus they may belong to the same minimal system of generators of $I_{G}$, contradicting Proposition 3.2. Thus there is no other generator of the form $B_{w^{\prime}}$ where $w^{\prime}$ is not an even cycle, except for $B_{w}$. 
The proof of the Theorem 5.4 shows that in the case that the complete intersection graph has two non-bipartite contiguous blocks the odd chordless cycle $c_{1}$ passing through $y_{1,2}$ is a unique cycle with this property. The same is true for the cycle $c_{2}$.

For a block $\mathcal{B}$, we denote by $I_{\mathcal{B}}$ the toric ideal $I_{G} \cap K\left[e_{i} \mid e_{i} \in \mathcal{B}\right]$, see [22].

The following result describes when a toric ideal $I_{G}$ is a complete intersection.

Theorem 5.5 Let $G$ be a graph and let $\mathcal{B}_{1}, \ldots, \mathcal{B}_{k}$ be its blocks. $I_{G}$ is a complete intersection toric ideal if and only if

(i) All minimal generators, except for at most one, of $I_{G}$ are of the form $B_{w}$ where $w$ is an even cycle and

(ii) The ideals $I_{\mathcal{B}_{i}}$ are complete intersection toric ideals for all $1 \leq i \leq k$.

Proof Let $G$ be a graph such that the toric ideal $I_{G}$ is a complete intersection. The first condition follows from Theorem 5.4 and the second from Theorem 3.1 by choosing $H=V\left(\mathcal{B}_{i}\right)$.

Conversely, let $G$ be a graph and let $\mathcal{B}_{1}, \ldots, \mathcal{B}_{k}$ be its blocks such that $I_{\mathcal{B}_{i}}$ is a complete intersection toric ideal for all $1 \leq i \leq k$. Note that every even cycle belongs to a unique block and all generators of the ideals $I_{\mathcal{B}_{i}}$ correspond to even cycles, see Theorem 5.3. The number of minimal generators of the block $\mathcal{B}_{i}$ is $m_{i}-n_{i}+1$ if $\mathcal{B}_{i}$ is bipartite and $m_{i}-n_{i}$ if not. Therefore, the total number of minimal generators of $I_{G}$ of the form $B_{w}$, where $w$ is an even cycle is

$$
\sum_{i=1}^{k}\left(m_{i}-n_{i}+1\right)-j,
$$

where $j$ is the number of non-bipartite blocks. Note that $\sum_{i=1}^{k} m_{i}=m$ since every edge belongs to a unique block. Also $\sum_{i=1}^{k} n_{i}=n+\sum_{i=1}^{c}\left(\operatorname{deg}\left(v_{i}\right)-1\right)$, where $v_{i}$ are cut vertices, $\operatorname{deg}\left(v_{i}\right)$ is the degree of $v_{i}$ as a vertex in the block tree $B(G)$ and $c$ is the number of cut vertices, since each cut vertex $v_{i}$ belongs to $\operatorname{deg}\left(v_{i}\right)$ blocks. The block tree $B(G)$ is bipartite with bipartition $(\mathbb{B}, \mathbb{S})$, where $\mathbb{B}$ is the set of blocks of $G$ and $\mathbb{S}$ is the set of cut vertices of $G$. Therefore, $\sum_{i=1}^{c} \operatorname{deg}\left(v_{i}\right)$ is the number of edges of the tree $B(G)$ which is $k+c-1$. Combining all these facts, we have that the total number of minimal generators of $I_{G}$ of the form $B_{w}$, where $w$ is an even cycle, is $m-n-\sum_{i=1}^{c} \operatorname{deg}\left(v_{i}\right)+c+k-j=m-n+1-j$.

We consider the following cases:

In the case $j=0$, the graph is bipartite and the total number of generators is $m-n+1$ since all minimal generators of $I_{G}$ are of the form $B_{w}$, where $w$ is an even cycle. This means that $G$ is a complete intersection.

In the case $j=1$, the graph is not bipartite and the total number of generators is $m-n$ since all minimal generators are of the form $B_{w}$, where $w$ is an even cycle. Therefore, $G$ is a complete intersection.

In the case $j=2$, the graph is not bipartite, thus its height is $m-n$ and the number of the minimal generators of the form $B_{w}$, where $w$ is an even cycle, is $m-n-1$ so there must be exactly one more which is not of that form from condition (i) and thus the total number of minimal generators is $m-n$ and $G$ is a complete intersection. 
In the case $j>2$, the graph is not bipartite, thus its height is $m-n$ and the number of the minimal generators of the form $B_{w}$, where $w$ is an even cycle, is $m-n+1-j$ so there must be exactly one more which is not of that form from condition (i) and thus the total number of minimal generators is $m-n+2-j$, which is less than the height, a contradiction to the generalized Krull's principal ideal theorem.

Therefore, in all possible cases, $G$ is a complete intersection.

Theorems 4.2 and 5.5, combined with the result of I. Gitler, E. Reyes, and R. Villarreal $[5,6]$ that the bipartite complete intersections graphs are the ring graphs, give the following corollary.

Corollary 5.6 Let $G$ be a graph. If $G$ is a complete intersection then either

(i) All blocks of $G$ are bipartite ring graphs, or

(ii) All blocks are bipartite ring graphs except for one, or

(iii) All blocks are bipartite ring graphs except for two which are contiguous.

This result characterizes almost completely, except of at most two blocks, the complete intersection graphs. In the next section, we will give properties of those one or two blocks which are not bipartite ring graphs.

\section{The odd cycle condition and normality}

In this section, we present Theorems 6.3 and 6.4 that are interesting on their own since they give us information about complete intersection graphs. But also they can be used to provide a necessary and sufficient condition for the edge ring of a complete intersection graph to be normal, see Theorem 6.7. The normalization of the edge subring $K[G]$ was described explicitly by A. Simis, W.V. Vasconcelos and R.V. Villarreal in [20] and by H. Ohsugi and T. Hibi in [15]. H. Ohsugi and T. Hibi related the normality of $K[G]$ with the odd cycle condition.

Definition 6.1 We say that a graph $G$ satisfies the odd cycle condition if for arbitrary two odd chordless cycles $c_{1}$ and $c_{2}$ in $G$, either $c_{1}, c_{2}$ have a common vertex or there exists an edge of $G$ joining a vertex of $c_{1}$ with a vertex of $c_{2}$.

For information about graphs satisfying the odd cycle condition, see $[9,16]$, and [21].

Theorem 6.2 (H. Ohsugi and T. Hibi [15]) Let $G$ be a graph. Then the following conditions are equivalent:

- The edge ring $K[G]$ is normal;

- The graph $G$ satisfies the odd cycle condition.

Theorem 6.3 Let $G$ be a biconnected complete intersection graph $G$. The graph $G$ satisfies the odd cycle condition and so the edge ring $K[G]$ is always normal. 
Proof Let $c_{1}, c_{2}$ be two odd chordless cycles of $G$ which have no common vertex. We will prove that the subgraph $\mathbf{c}_{1} \cup \mathbf{c}_{2}$ has a chord. Suppose not. Let $p=$ $\left(y_{1}, x_{1}, \ldots, x_{2}, y_{2}\right)$ be the shortest path from $c_{1}$ to $c_{2}$, denoted by its vertices. The length of $p$ is greater than one, so it may be that $x_{1}=x_{2}$. The subgraph $\mathbf{c}_{1} \cup \mathbf{c}_{2} \cup \mathbf{p}$ has chords, otherwise the walk $\left(c_{1}, p, c_{2},-p\right)$ defines a minimal generator, which contradicts Theorem 5.3. Since $p$ is the shortest path, it is chordless and there is no chord in $\mathbf{c}_{1} \cup \mathbf{c}_{2}$. Therefore, all the chords of $\mathbf{c}_{1} \cup \mathbf{c}_{2} \cup \mathbf{p}$ should be from the cycle $c_{1}$ to $x_{1}$ and from the cycle $c_{2}$ to $x_{2}$. Let $\left(u_{1,1}, u_{1,2}, \ldots, u_{1, s_{1}}\right)$ be the cycle $c_{1}$ denoted by its vertices, where $u_{1,1}=y_{1}$. We consider all chords from the cycle $c_{1}$ to $x_{1}$ together with the edge $\left\{x_{1}, y_{1}\right\}$ and denote them by $e_{1}=$ $\left\{x_{1}, u_{1, i_{1}}\right\}, \ldots, e_{t_{1}}=\left\{x_{1}, u_{1, i_{t_{1}}}\right\}$, where $1=i_{1}<\cdots<i_{t_{1}}$. In the case that $t_{1}>1$, the cycles $c_{1, j}=\left(x_{1}, u_{1, i_{j}}, u_{1, i_{j}+1}, \ldots, u_{1, i_{j+1}}\right)$, for $1 \leq j \leq t_{1}-1$, and $c_{1, t_{1}}=$ $\left(x_{1}, u_{1, i_{t_{1}}}, u_{1, i_{t_{1}}+1}, \ldots, u_{1, s_{1}}, u_{1,1}\right)$ are chordless and at least one of them is odd, say $c_{1, j}$, since $c_{1}$ is odd. Similarly, if $t_{2}>1$ there must be an odd chordless cycle of the form $c_{2, k}$.

In the case $t_{1}>1$ and $t_{2}>1$, let $w$ be the even closed path $\left(c_{1, j}, x_{1}, \ldots, x_{2}, c_{2, k}\right)$.

In the case $t_{1}=1$ and $t_{2}>1$, let $w$ be the even closed path $\left(c_{1}, y_{1}, x_{1}, \ldots, x_{2}, c_{2, k}\right)$.

In the case $t_{1}>1$ and $t_{2}=1$, let $w$ be the even closed path $\left(c_{1, j}, x_{1}, \ldots, x_{2}, y_{2}, c_{2}\right)$.

In the case $t_{1}=1$ and $t_{2}=1$, let $w$ be the even closed path $\left(c_{1}, y_{1}, x_{1}, \ldots, x_{2}\right.$, $\left.y_{2}, c_{2}\right)$.

In all cases $w$ is chordless; therefore, by Theorem 2.7, the binomial $B_{w}$ is a minimal generator of $I_{G}$, contradicting Theorem 5.3. We conclude that $G$ satisfies the odd cycle condition.

Theorem 6.4 Let $G$ be a complete intersection graph such that it contains two nonbipartite blocks $\mathcal{B}_{1}, \mathcal{B}_{2}$. Both $\mathcal{B}_{1}, \mathcal{B}_{2}$ contain exactly one odd chordless cycle passing through the cut point $y_{1,2}$ and $y_{2,1}$, respectively. If any of them contained another odd chordless cycle then this cycle would have distance one from the cut point $y_{1,2}$ if it is in $\mathcal{B}_{1}$ or $y_{2,1}$ if it is in $\mathcal{B}_{2}$.

Proof Let $G$ be a complete intersection graph such that it contains two nonbipartite blocks $\mathcal{B}_{1}, \mathcal{B}_{2}$. The two blocks are contiguous, see Theorem 4.2. According to the proof of Theorem 4.2, there is one generator of the form $B_{w}$, where $w=\left(c_{1}, p, c_{2},-p\right)$ is an even closed walk, where $c_{1}, c_{2}$ are the odd chordless cycles of $\mathcal{B}_{1}, \mathcal{B}_{2}$ which are passing through $y_{1,2}$ and $y_{2,1}$, respectively, and $p$ is the unique chordless path between them. Let $c_{1}=\left(\left\{y_{1,2}, y_{1}\right\}, \xi_{1},\left\{y_{2}, y_{1,2}\right\}\right)$, where $\xi_{1}$ is a path from $y_{1}$ to $y_{2}$. Suppose that the block $\mathcal{B}_{1}$ contains another odd chordless cycle. Let $c$ be an odd chordless cycle different from $c_{1}$ and $\xi$ be a path of smallest length from $c$ to $y_{1,2}$. Look at the even closed walk $w^{\prime}=\left(c, \xi, p, c_{2},-p,-\xi\right)$. Then $B_{w^{\prime}}$ is not a minimal generator since it has a common cycle, $c_{2}$, with $w$ and $\operatorname{deg}_{G}\left(B_{w^{\prime}}\right) \neq \operatorname{deg}_{G}\left(B_{w}\right)$. Since $c, c_{2}$ are chordless cycles, $\xi$ and $p$ are chordless paths and $c, \xi$ belong to the block $\mathcal{B}_{1}, c_{2}$ belongs to the block $\mathcal{B}_{2}$ and each edge of $p$ belongs to a different block; therefore, there must be at least one chord (bridge of $w^{\prime}$ ), say $e$, from the cycle $c$ to the path $\xi$, see Theorem 2.7. And since $\xi$ is a path of smallest length from $c$ to $y_{1,2}$, any chord should be from $c$ to the second vertex of $\xi$, 
Fig. 3 A block of type $T_{4}$

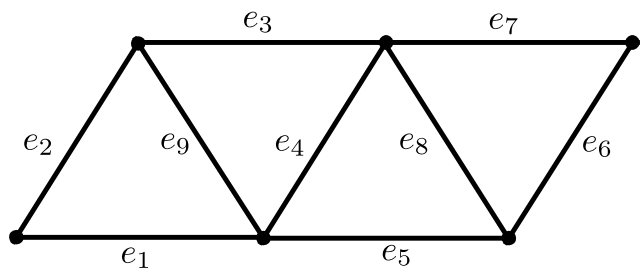

let it be $x$. So, certainly $c$ does not pass through $y_{1,2}$ and this implies that $c_{1}$ is the only odd chordless cycle which belongs to the block $\mathcal{B}_{1}$ which passes through $y_{1,2}$. Look at the induced graph of $\mathbf{c} \cup\{x\}$. Since $c$ is chordless, any chord of $\mathbf{c} \cup\{x\}$ is from $c$ to $x$. Let $\left(u_{1}, u_{2}, \ldots, u_{s}\right)$ be the cycle $c$ denoted by its vertices. We consider all chords from the cycle $c$ to $x$ and denote them by $e_{1}=\left\{x, u_{i_{1}}\right\}, \ldots, e_{t}=\left\{x, u_{i_{t}}\right\}$, where $i_{1}<\cdots<i_{t}$. Note that $t \geq 2$, since $e$ is a chord of $\mathbf{c} \cup\{x\}$ and the first edge of the path $\xi$ is also a chord of $\mathbf{c} \cup\{x\}$. The cycles $o_{j}=\left(x, u_{i_{j}}, u_{i_{j}+1}, \ldots, u_{i_{j+1}}, x\right)$, for $1 \leq j \leq t-1$, and $o_{t}=\left(x, u_{i_{t}}, u_{i_{t}+1}, \ldots, u_{s}, u_{1}, \ldots, u_{i_{1}}, x\right)$ are chordless and at least one of them is odd since $c$ is odd. Without loss of generality, we can suppose that it is $o_{1}$. But then, the even closed walk $w^{\prime \prime}=\left(o_{1}, \xi^{\prime}, p, c_{2},-p,-\xi^{\prime}\right)$ has no chords and bridges, where $\xi^{\prime}$ is the subpath of $\xi$ from $x$ to $y_{1,2}$. Therefore, by Theorem 2.7, $B_{w^{\prime \prime}}$ is a minimal generator of $I_{G}$. But this is not possible since $w^{\prime \prime}$ has a common cycle, $c_{2}$, with $w$, except if $B_{w^{\prime \prime}}=B_{w}$. Therefore, $o_{1}$ has to be $c_{1}, \xi^{\prime}=\varnothing, u_{i_{1}}=y_{1}$, $u_{i_{2}}=y_{2}$, and $x=y_{1,2}$. Therefore, $c$ has distance one from $y_{1,2}$.

Definition 6.5 We say that a block is of type $T_{i}$ if it has $i$ chordless odd cycles.

A bipartite block is of type $T_{0}$, while in a complete intersection graph the nonbipartite blocks may be of higher type than $T_{1}$. For example, the graph in Fig. 3 is of type $T_{4}$ and it is complete intersection, since $I_{G}=\left(e_{1} e_{3}-e_{2} e_{4}, e_{4} e_{6}-e_{5} e_{7}, e_{3} e_{5}-\right.$ $\left.e_{8} e_{9}\right)$ and $h=9-6=3$.

\section{Definition 6.6}

Two non-bipartite blocks are called strongly contiguous if

- Both are of type $T_{1}$ and they have distance at most one, or

- One is of type $T_{1}$ and the other of greater type and they have a common cut vertex.

It is easy to see that strongly contiguous blocks are always contiguous. The next theorem completely determines the form of the complete intersection graphs $G$ for which their edge ring $K[G]$ is normal.

Theorem 6.7 Let $G$ be a complete intersection graph. Then $G$ is normal if and only if $G$ has at most one non-bipartite block or two which are strongly contiguous.

Proof Let $G$ be a complete intersection graph such that $K[G]$ is normal, then $G$ satisfies the odd cycle property. From Theorem 4.2 we know that $G$ has at most one non-bipartite block or two which are contiguous. In the first case, we have nothing to prove. Suppose that we are in the case that there are two contiguous blocks $\mathcal{B}_{1}, \mathcal{B}_{2}$. 


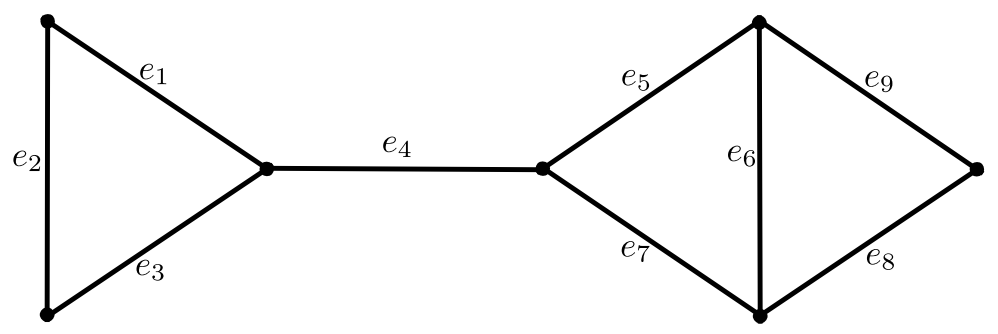

Fig. 4 The example

In the case that both are of type $T_{1}$, from Theorem 6.4, they have exactly one odd chordless cycle each, passing through $y_{1,2}$ and $y_{2,1}$, respectively. Since $G$ satisfies the odd cycle property, the two blocks $\mathcal{B}_{1}, \mathcal{B}_{2}$ have to have distance at most one.

In the case that one is of type $T_{1}$ and the other of greater type, say $\mathcal{B}_{1}$ is the first and $\mathcal{B}_{2}$ the second, the block $\mathcal{B}_{1}$ has only one odd chordless cycle passing through $y_{1,2}$, the block $\mathcal{B}_{2}$ has more than one chordless cycle, one is passing through $y_{2,1}$ and the others have distance one from $y_{2,1}$, by Theorem 6.4. Since $G$ satisfies the odd cycle property, the two blocks have to have a common (cut) vertex, $y_{1,2}=y_{2,1}$. Finally, it is impossible to have both $\mathcal{B}_{1}, \mathcal{B}_{2}$ of type greater than $T_{1}$, since in this case there is an odd chordless cycle in $\mathcal{B}_{1}$ with distance one from $y_{1,2}$ and there is an odd chordless cycle in $\mathcal{B}_{2}$ with distance one from $y_{2,1}$. So these two odd cycles have distance at least two, contradicting the odd cycle property. So in all possible cases, the two blocks are strongly contiguous.

For the converse, in the case that the graph $G$ has at most one non-bipartite block, Theorem 6.3 implies that $G$ satisfies the odd cycle property and thus $K[G]$ is normal. In the case that $G$ has two non-bipartite blocks which are strongly contiguous, Theorem 6.4 implies that $G$ satisfies the odd cycle condition and thus $K[G]$ is normal.

For example, consider the graph $G$ in Fig. 4 with seven vertices $v_{1}, v_{2}, \ldots, v_{7}$ and nine edges $e_{1}=\left\{v_{1}, v_{2}\right\}, e_{2}=\left\{v_{2}, v_{3}\right\}, e_{3}=\left\{v_{3}, v_{1}\right\}, e_{4}=\left\{v_{1}, v_{4}\right\}, e_{5}=\left\{v_{4}, v_{5}\right\}$, $e_{6}=\left\{v_{5}, v_{6}\right\}, e_{7}=\left\{v_{6}, v_{4}\right\}, e_{8}=\left\{v_{6}, v_{7}\right\}, e_{9}=\left\{v_{5}, v_{7}\right\}$. The graph $G$ has two nonbipartite blocks which are contiguous. The one is of type $T_{1}$ and the other of type $T_{2}$ and they have distance one. Therefore, they are not strongly contiguous. From Theorem 5.5, the graph $G$ is a complete intersection graph and the toric ideal is generated by the two circuits $e_{1} e_{3} e_{5} e_{7}-e_{2} e_{4}^{2} e_{6}$ and $e_{5} e_{8}-e_{7} e_{9}$. It follows from Theorem 6.7 that the edge ring $K[G]$ is not normal, but note that the ideal $I_{G}$ is generated by circuits with a square-free term, see Theorem 3.2 of [13].

Acknowledgement The authors would like to thank the referees for their valuable comments that helped improve the article.

\section{References}

1. Bermejo, I., Garcia-Marco, I., Reyes, E.: Complete intersection graphs. arXiv:1210.1950v1

2. Bogart, T., Jensen, A.N., Thomas, R.R.: The circuit ideal of a vector configuration. J. Algebra 309, 518-542 (2007) 
3. Charalambous, H., Katsabekis, A., Thoma, A.: Minimal systems of binomial generators and the indispensable complex of a toric ideal. Proc. Am. Math. Soc. 135, 3443-3451 (2007)

4. Doering, L., Gunston, T.: Algebras arising from planar bipartite graphs. Commun. Algebra 24, 35893598 (1996)

5. Gitler, I., Reyes, E., Villarreal, R.: Ring graphs and toric ideals. Electron. Notes Discrete Math. 28C, 393-400 (2007)

6. Gitler, I., Reyes, E., Villarreal, R.: Ring graphs and complete intersection toric ideals. Discrete Math. 310, 430-441 (2010)

7. Fischer, K., Morris, W., Shapiro, J.: Affine semigroup rings that are complete intersections. Proc. Am. Math. Soc. 125, 3137-3145 (1997)

8. Fischer, K., Shapiro, J.: Mixed matrices and binomial ideals. J. Pure Appl. Algebra 113, 39-54 (1996)

9. Fulkerson, D.R., Hoffman, A.J., McAndrew, M.H.: Some properties of graphs with multiple edges. Can. J. Math. 17, 166-177 (1965)

10. Herzog, J.: Generators and relations of abelian semigroups and semigroup rings. Manuscr. Math. 3, 175-193 (1970)

11. Katzman, M.: Bipartite graphs whose edge algebras are complete intersections. J. Algebra 220, 519530 (1999)

12. Kuratowski, K.: Sur le problème des courbes gauches en topologie. Fundam. Math. 15, 271-283 (1930)

13. Martinez-Bernal, J., Villarreal, R.H.: Toric ideals generated by circuits. Algebra Colloq. 19, 665-672 (2012)

14. Morales, M., Thoma, A.: Complete intersections lattice ideals. J. Algebra 284, 755-770 (2005)

15. Ohsugi, H., Hibi, T.: Normal polytopes arising from finite graphs. J. Algebra 207, 409-426 (1998)

16. Ohsugi, H., Hibi, T.: Indispensable binomials of finite graphs. J. Algebra Appl. 4(4), 421-434 (2005)

17. Reyes, E., Tatakis, Ch., Thoma, A.: Minimal generators of toric ideals of graphs. Adv. Appl. Math. 48(1), 64-78 (2012)

18. Scheja, G., Scheja, O., Storch, U.: On regular sequences of binomials. Manuscr. Math. 98, 115-132 (1999)

19. Simis, A.: On the Jacobian module associated to a graph. Proc. Am. Math. Soc. 126, 989-997 (1998)

20. Simis, A., Vasconcelos, W.V., Villarreal, R.H.: The integral closure of subrings associated to graphs. J. Algebra 199, 281-289 (1998)

21. Stanley, R.P.: A zonotope associated with graphical degree sequences. In: Gritzmann, P., Sturmfels, B. (eds.) Applied Geometry and Discrete Mathematics. DIMACS Ser. Discrete Math. Theoret. Comput. Sci., vol. 4, pp. 555-570. Am. Math. Soc., Providence (1991)

22. Sturmfels, B.: Gröbner Bases and Convex Polytopes. University Lecture Series, vol. 8. Am. Math. Soc., Providence (1995)

23. Villarreal, R.H.: Rees algebras of edge ideals. Commun. Algebra 23, 3513-3524 (1995) 